\title{
Benefit-Cost Ratios of Continuing Routine Immunization During the COVID-19 Pandemic in Africa
}

\author{
Elizabeth Watts ${ }^{1,2,3} *$ (D), Joshua $\mathrm{Mak}^{2,3}$ and Bryan Patenaude ${ }^{2,3}$ \\ ${ }^{1}$ Division of Health Policy and Management, School of Public Health, University of Minnesota, Minneapolis, \\ MN, USA \\ ${ }^{2}$ International Vaccine Access Center, Johns Hopkins Bloomberg School of Public Health, Baltimore, MD, USA \\ ${ }^{3}$ Department of International Health, Johns Hopkins Bloomberg School of Public Health, Baltimore, MD, USA \\ *Corresponding author: e-mail: watts229@umn.edu
}

Keywords: benefit-cost analysis, COVID-19, routine immunization, vaccines

JEL classifications: D61, I18, N37

\begin{abstract}
Disruptions in routine immunization caused by COVID-19 put African countries with large vaccinepreventable disease burdens at high risk of outbreaks. Abbas et al. (2020) showed that mortality reduction from resuming immunization outweighs excess mortality from COVID-19 caused by exposure during immunization activities. We leverage these estimates to calculate benefit-cost ratios (BCRs) of disrupted immunization and apply cost of illness (COI) and value of statistical life-year (VSLY) approaches to estimate the cost of excess child deaths from eight vaccine-preventable diseases. BCRs were computed for each country, vaccine, and Expanded Program on Immunization visit. Secondary estimates that include the cost of providing immunization are presented in scenario analysis. Suspended immunization may cost $\$ 4949$ million due to excess mortality using the COI approach, or $\$ 34,344$ million using the VSLY approach. Likewise, excess COVID-19 deaths caused by exposure from immunization activities would cost $\$ 53$ and $\$ 275$ million using the COI and VSLY approaches, respectively. BCRs of continuing routine immunization are 94:1 using COI and 125:1 using VSLY, indicating that the economic costs of suspending immunization exceed that of COVID19 deaths risked by routine immunization. When including the costs of providing routine immunization during the COVID-19 pandemic, the BCRs are 38:1 and 97:1 using the COI and VSLY approaches, respectively.
\end{abstract}

\section{Introduction}

Egypt's first COVID-19 cases were reported on 14 February 2020 (World Health Organization, 2020a). Twenty-six days later, the World Health Organization (WHO) declared COVID-19 a pandemic (World Health Organization, 2020e). By 7 April 2020, COVID-19 cases have been detected in 52 African countries (World Health Organization, 
2020a). The COVID-19 pandemic has disrupted health systems' abilities to provide basic services worldwide (World Health Organization, 2020c). Based on a survey by the WHO conducted between May and July 2020, 50 \% of responding countries have experienced disruptions to immunization services, with severe disruptions more common in low- and middle-income countries (LMICs) (World Health Organization, 2020c).

Immunization services play an important role in disease control and are responsible for preventing thousands of child deaths each year (Andre et al., 2008; Li et al., 2019). Prior to the COVID-19 pandemic, the return on investment for vaccines was estimated to range from \$21 to \$54 in LMICs (Sim et al., 2020). Although vaccine coverage has improved in African countries, rates remain uneven within and between countries (Mosser et al., 2019). Efforts to increase or maintain immunization coverage in 2020 have been thwarted by measures aimed at slowing the spread of COVID-19, such as physical distancing and travel restrictions (World Health Organization, 2020d). Disruptions in service stem from supply- and demandside factors, including supply chain disruptions, health facility closures, transportation challenges during lockdowns, and fear of contracting COVID-19 (World Health Organization, 2020c). Backslides in immunization coverage will exacerbate existing inequities in immunization coverage and disease burden (World Health Organization and UNICEF, 2020).

In facing the ongoing challenge of continuing health services during the COVID-19 pandemic, countries must prioritize and adapt services to comply with COVID-19 prevention measures (World Health Organization, 2020b). During vaccination visits, close contact between healthcare workers, children, and caregivers may contribute to increased COVID19 transmission, whereas safety protocols to reduce COVID-19 transmission may increase costs of providing immunization services. Understanding the health and economic benefits and risks of continuing immunization services during the COVID-19 pandemic may help decision-makers make informed choices about service prioritization.

A recent study examined the health benefits and risks associated with continuing routine immunization activities in Africa during the COVID-19 lockdown (Abbas et al., 2020). The epidemiologic model estimated the benefits and risks of a 6-month suspension of routine immunization services during the COVID-19 pandemic in African countries. Excess childhood deaths due to disrupted childhood immunizations were compared to excess COVID-19 fatalities attributable to people engaging in routine immunization activities. Benefits of immunization were included for 11 vaccine-preventable pathogens: diphtheria, tetanus, pertussis, hepatitis B, Haemophilus influenzae type b, Streptococcus pneumoniae, rotavirus, measles, rubella, Neisseria meningitidis serogroup A (MenA), and yellow fever. Abbas et al. also estimated the risk of COVID-19 fatalities for the vaccinated children and members of their households: siblings, parents, and grandparents. They found that the lives saved from continuing routine immunization services would exceed household COVID-19 fatalities at a ratio of 84:1 (95\% confidence interval: 14-267), on average, across all African countries (Abbas et al., 2020).

To contextualize the economic benefits and risks of continuing immunization services in African countries during the COVID-19 pandemic, estimates of lives saved from vaccinepreventable diseases and deaths from COVID-19 attributable to immunization activities (Abbas et al., 2020) were used to calculate benefit-cost ratios (BCRs). Furthermore, benefits of immunization may only be realized if sufficient investment in immunization is made. A secondary analysis leverages recent estimates of the costs of immunization to assess whether the economic benefits of lives saved by immunization exceed costs of providing immunization during the COVID-19 pandemic and risks of excess COVID-19 deaths. 


\section{Materials and methods}

Costs of excess deaths caused by a 6-month suspension of routine immunization services were estimated for eight vaccines in all 54 African countries (Appendix 1 in the Supplementary Material). Since multiple vaccines are given simultaneously, the BCRs were calculated independently for each pathogen and per vaccination visit. Hepatitis B, $H$. influenzae type b, and S. pneumoniae are designated as Expanded Program on Immunization (EPI) visit 1 (EPI-1); EPI-2 consists of rotavirus, measles first dose, rubella, N. meningitidis serogroup A (MenA), and yellow fever vaccine (YFV); EPI-3 is only for measles second dose. The economic benefits of lives saved were compared to costs of excess COVID-19 deaths attributable to transmission during routine immunization.

Two approaches were used to estimate the economic costs of vaccine-preventable deaths and COVID-19 deaths. The first approach was the cost of illness (COI) approach, which includes direct costs (treatment and transportation costs) and indirect costs (caregiver wages lost and productivity loss due to death). The second approach was the value of statistical lifeyear (VSLY) approach, which reflects the marginal rate of substitution between goods and one additional year of life (Robinson et al., 2019a). When VSLY is multiplied over the life expectancy, it can be interpreted as the willingness to pay to reduce risk of death, adjusted for age (Robinson et al., 2019a). For each approach, the estimated economic benefits of lives saved by routine immunization were divided by the costs of excess deaths from COVID-19 to compute BCRs. All costs are reported in 2018 USD and reflect economic costs incurred in the year 2020 .

\subsection{Cost of illness (vaccine-preventable diseases)}

Estimates of vaccine-preventable child deaths from a 6-month suspension of immunization (Abbas et al., 2020) were used to estimate treatment costs, transportation costs, lost caregiver wages, and productivity loss due to death. Since deaths from vaccine-preventable diseases (VPDs) occur 1-5 years after missed vaccinations, costs were discounted from the year of death to the year 2020 at $3 \%$ per year.

Direct costs include treatment and transportation costs from illness. Descriptive statistics of inputs for the approach are provided in Appendix 2 in the Supplementary Material. Treatment costs averted were estimated by multiplying the number of cases by syndromespecific care-seeking rates (Demographic and Health Survey Program, n.d.). Although only fatal cases were modeled, demand and supply side barriers exacerbated by the COVID-19 pandemic, including fear of contracting the coronavirus, transportation restrictions, and health facility closures (World Health Organization and UNICEF, 2020), may prevent some severe and fatal cases from reaching health facilities. To account for this uncertainty, careseeking rates were varied in sensitivity analysis. We assumed all care-seeking cases would be admitted to inpatient care. The number of care-seeking cases was multiplied by the syndrome-specific length of stay (Parashar et al., 2003; Rudan et al., 2004; Sinha et al., 2008; Kim et al., 2010a; Bishai et al., 2011; Lee et al., 2011) and the per diem inpatient health facility cost (Stenberg et al., 2018). Costs of medications and diagnostics were conservatively estimated to be 25-50 \% of the facility fees (de Broucker et al., 2020). Transportation costs were estimated by multiplying the cost per round trip to a healthcare facility (Kim et al., 2010b) by the estimated number of care-seeking cases.

Caregiver wages lost were estimated using minimum wage (U.S. Department of State, 2017; International Labor Organization, 2018) as a proxy for the opportunity cost of caring 
for a sick child, as some caregivers may not be formally employed or may have the lowest opportunity cost of time in the household. Lost caregiver wages were estimated by multiplying the wage by the number of care-seeking cases and length of stay (Parashar et al., 2003; Rudan et al., 2004; Sinha et al., 2008; Kim et al., 2010a; Bishai et al., 2011; Lee et al., 2011).

We used the human capital approach to estimate productivity loss due to death. Future productivity loss from vaccine-preventable deaths was estimated for years of life lost between ages 15 and 64 . The number of productive years lost was multiplied by the gross domestic product (GDP) per capita in 2018 (The World Bank, 2019) to approximate an individual's economic contribution to society. Since all deaths from VPDs occurred before reaching the working age, we adjusted the number of deaths by the probability of survival to 15 years and discounted to 2020 at $3 \%$ per year (The United Nations, 2019). Productivity loss was multiplied by the country-specific labor force participation rate to account for individuals who would not have participated in the labor market (International Labor Organization, 2018).

\subsection{Cost of illness (COVID-19)}

We used a similar approach to estimate the COI associated with COVID-19 deaths attributable to routine immunization activities. Costs associated with COVID-19 deaths were not discounted, because they were assumed to occur in 2020. The care-seeking rate for childhood pneumonia (Demographic and Health Survey Program, n.d.) was applied as a proxy for the proportion of COVID-19 cases that would seek care and varied the rate in sensitivity analysis. All care-seeking cases were assumed to be admitted for inpatient treatment. Evidence measuring COVID-19 treatment costs in inpatient and intensive care unit (ICU) settings was available for Kenya (Barasa \& Kairu, 2020). These costs were converted to 2018 USD and adjusted to the remaining African countries using the ratio of modeled inpatient facility costs (Stenberg et al., 2018) in the target countries compared to Kenya. We assumed that all patients would initially receive treatment in a standard inpatient setting for 5 days and half of the patients would be transferred to an intensive care setting for an additional 7 days (Rees et al., 2020). In middle- and high-income countries, most COVID-19 deaths occur in an ICU setting (Wang et al., 2020). However, availability and access to intensive care settings in African countries may be limited especially during the COVID-19 pandemic (Davies et al., 2020; Ma \& Vervoort, 2020). To account for this uncertainty, we varied the rate of intensive care transfers in sensitivity analysis (Appendix 3 in the Supplementary Material).

As for VPD cases, transportation costs were calculated by multiplying the number of care-seeking cases by the cost of one round trip to a health facility. Lost caregiver wages were estimated by multiplying the minimum wage by the length of stay.

Productivity loss due to death from COVID-19 was estimated by multiplying GDP per capita by remaining productive years at the age of death. To align with the epidemiologic study, we assumed that COVID-19 deaths may occur in individuals in four different age groups: the vaccinated child (age 1), a sibling of the vaccinated child (age 5), parents of the vaccinated child, and grandparents (Abbas et al., 2020). To estimate the age at death among parents of the vaccinated child, we used the country-specific average childbearing age; for grandparents, we used twice the average childbearing age (The United Nations, 2017). The number of remaining productive years was calculated for each age group by taking the retirement age minus the age at death, or the retirement age minus age 15 for children. 
Additionally, for vaccinated children and their siblings, we adjusted the number of predicted COVID-19 deaths by the probability of survival to age 15 (The United Nations, 2017). For each group, remaining productive life years were multiplied by the GDP per capita and discounted to 2020 at $3 \%$ per year. We calculated a weighted average of the productivity loss associated with COVID-19 deaths using the country-specific proportions of COVID-19 deaths at each age. Total productivity loss was estimated by multiplying the weighted average by the number of COVID-19 deaths and the labor force participation rate (International Labor Organization, 2018).

\subsection{Value of statistical life year}

Value of statistical life (VSL) is a risk assessment metric based on estimates of the average willingness to pay for a small reduction in mortality risk in a population (Robinson et al., 2019a). The marginal willingness to pay is typically multiplied across a population to estimate the value society places on saving one life (Robinson et al., 2019a). Using the standard approach, VSL does not vary based on the age of those whose lives are saved; thus, it does not provide additional insight beyond the benefit-risk ratios that compare the number of VPD deaths to that of COVID-19 deaths. Since our model only includes children, and COVID-19 disproportionately impacts older adults, we applied the VSLY approach. VSLY is derived from VSL and reflects the marginal rate of substitution between gains in life expectancy and other goods and services.

We first estimated VSL using methods outlined in the Reference Case Guidelines for Benefit-Cost Analysis in Global Health and Development (Robinson et al., 2019a). Since few direct estimates of VSL were available for the modeled countries, we applied a valuetransfer approach to extrapolate VSL from the USA to African countries. The value-transfer approach adjusts for differences in income using a multiplier of the ratio of GDP per capita in the target country and the USA raised to the income elasticity for health. For this analysis, we assumed an income elasticity of 1.5 as estimated in a recent systematic review (Robinson et al., 2019b).

Deaths caused by the VPDs included in the analysis are estimated to occur within the next 5 years, so we estimated VSL using projected GDP per capita in the year of death (Global Burden of Disease Health Financing Collaborator Network, 2018). We applied the formula below, in which $i$ indicates the target country, the USA serves as the reference country, $j$ is the target year, and $e_{y}$ is the income elasticity. The U.S. VSL was estimated as 160 times GDP per capita (The World Bank, 2019; Robinson et al., 2019a), and was approximately $\$ 10$ million in 2018. For countries for which this method yields VSLs below the estimated value of future earnings, or 20 times GDP per capita, we imputed 20 times GDP per capita as a minimum value for the VSL (Robinson et al., 2019a).

$$
\operatorname{VSL}_{i, j}=\left(\frac{\text { GDP per capita }_{i, j}}{\text { GDP per capita }_{\mathrm{USA}, j}}\right)^{e_{y}} \times \mathrm{VSL}_{\mathrm{USA}, j} .
$$

We derived VSLY by dividing VSL by the country-specific average remaining life expectancy, which was approximated by taking half of each country's undiscounted life expectancy at birth. We then multiplied the VSLY by the expected number of remaining life years at the age of death. For child deaths, this yields a higher value than the standard VSL approach; for older adults, it yields a lower value. 


\subsection{Benefit-cost ratios}

To compute BCRs, we divided economic benefits of sustained immunization by costs of COVID-19 deaths attributable to immunization activities. BCRs using the COI and VSLY approaches were estimated for each country, vaccine, and EPI visit. The primary results are based on the calculation below:

$$
\mathrm{BCR}=\frac{(\text { Benefits of lives saved by routine immunization })}{(\text { Costs of COVID }-19 \text { deaths from interaction during immunization visits })} .
$$

\subsection{Scenario analysis}

The primary analysis compares the economic benefits of lives saved from routine immunization to the economic costs of additional COVID-19 deaths due to interaction during immunization visits. Because the full benefits of immunization would only be realized if investment in providing immunization is continued, we conducted scenario analysis to include the cost of routine immunization in the BCR. Routine immunization costs in African countries for the year 2021 were extracted from an analysis of the costs of reaching coverage goals outlined in the Immunization Agenda 2030 (Sriudomporn et al., 2021). Annual routine immunization costs were adjusted to 2020 using a ratio of the target population (United Nations, 2019) in 2020-2021. We divided these costs by 2 to approximate the cost of immunization during the 6-month suspension in the model. Furthermore, providing routine immunization during the COVID-19 pandemic may be more costly with mitigation measures in place. A recent study estimated that providing personal protective equipment, implementing social distancing, providing hazard pay, and implementing other mitigation measures may increase the cost per dose by 23-251\% depending on the setting and volume of the vaccination site (Banks et al., 2021). We estimated BCRs that include the cost of providing routine immunization at 100,123 , and $351 \%$ of the baseline costs in addition to the cost of COVID-19 deaths estimated. The BCR calculation for the scenario analyses is below:

$$
\mathrm{BCR}=\frac{\text { (Benefits of lives saved by routine immunization })}{(\text { Costs of COVID }-19 \text { deaths }+ \text { Cost of routine immunization })} \text {. }
$$

\subsection{Sensitivity analysis}

We selected five key parameters to vary in probabilistic sensitivity analysis: number of deaths from VPDs and COVID-19, care-seeking rates, ICU admittance rate for COVID-19 patients, facility costs, and GDP per capita (Appendix 4 in the Supplementary Material). Sensitivity analysis for VSLY was performed by varying GDP per capita and the number of deaths, as the other inputs do not impact VSLY calculations. We generated 10,000 Monte Carlo simulations to produce $95 \%$ confidence intervals for all estimates.

We used one-way sensitivity analysis to assess the impact each parameter on the results by estimating benefits, costs, and BCRs within two standard deviations of the mean value for each of the five parameters listed above.

\section{Results}

Using the COI approach, we estimated that sustaining immunization could avert $\$ 4949$ million (\$4776-5480) from costs associated with vaccine-preventable child deaths in the next 5 years (Table 1). Treatment costs, transportation costs, and caregiver wages together 
Table 1. Benefits, risks, and BCRs of sustained routine immunization in Africa using the COI and VSLY approaches (95 \% confidence intervals in parentheses).

\begin{tabular}{|c|c|c|c|c|c|c|c|}
\hline \multirow[b]{2}{*}{ Vaccine } & \multirow{2}{*}{$\begin{array}{c}\text { Benefit-risk } \\
\text { ratio } \\
\text { (VPD deaths: } \\
\text { COVID-19 } \\
\text { deaths) }\end{array}$} & \multicolumn{3}{|c|}{ COI } & \multicolumn{3}{|c|}{ VSLY } \\
\hline & & $\begin{array}{c}\text { VPD } \\
\text { (millions) }\end{array}$ & $\begin{array}{l}\text { COVID-19 } \\
\text { (millions) }\end{array}$ & BCR (COI) & VPD (millions) & $\begin{array}{l}\text { COVID-19 } \\
\text { (millions) }\end{array}$ & $\begin{array}{c}\text { BCR } \\
(\mathrm{VSLY})\end{array}$ \\
\hline HepB3 & $\begin{array}{c}1 \\
(0-2)\end{array}$ & $\begin{array}{c}\$ 51 \\
(41-107)\end{array}$ & $\begin{array}{c}\$ 34 \\
(33-80)\end{array}$ & $\begin{array}{c}1 \\
(1-2)\end{array}$ & $\begin{array}{c}\$ 357 \\
(273-743)\end{array}$ & $\begin{array}{c}\$ 178 \\
(150-323)\end{array}$ & $\begin{array}{c}2 \\
(1-4)\end{array}$ \\
\hline Hib3 & $\begin{array}{c}9 \\
(1-31)\end{array}$ & $\begin{array}{c}\$ 738 \\
(677-856)\end{array}$ & $\begin{array}{c}\$ 36 \\
(33-80)\end{array}$ & $\begin{array}{c}21 \\
(9-23)\end{array}$ & $\begin{array}{c}\$ 5242 \\
(4696-6138)\end{array}$ & $\begin{array}{c}\$ 178 \\
(150-325)\end{array}$ & $\begin{array}{c}29 \\
(16-37)\end{array}$ \\
\hline PCV3 & $\begin{array}{c}10 \\
(5-13)\end{array}$ & $\begin{array}{c}\$ 637 \\
(560-800)\end{array}$ & $\begin{array}{c}\$ 30 \\
(28-73)\end{array}$ & $\begin{array}{c}21 \\
(9-25)\end{array}$ & $\begin{array}{c}\$ 4499 \\
(3841-5768)\end{array}$ & $\begin{array}{c}\$ 155 \\
(125-290)\end{array}$ & $\begin{array}{c}29 \\
(15-39)\end{array}$ \\
\hline RotaC & $\begin{array}{c}4 \\
(1-14)\end{array}$ & $\begin{array}{c}\$ 170 \\
(149-205)\end{array}$ & $\begin{array}{c}\$ 15 \\
(14-38)\end{array}$ & $\begin{array}{c}11 \\
(4-13)\end{array}$ & $\begin{array}{c}\$ 1133 \\
(968-1397)\end{array}$ & $\begin{array}{c}\$ 70 \\
(56-139)\end{array}$ & $\begin{array}{c}16 \\
(8-22)\end{array}$ \\
\hline MCV1 & $\begin{array}{c}99 \\
(14-327)\end{array}$ & $\begin{array}{c}\$ 2994 \\
(2754-5364)\end{array}$ & $\begin{array}{c}\$ 12 \\
(11-28)\end{array}$ & $\begin{array}{c}257 \\
(107-257)\end{array}$ & $\begin{array}{c}\$ 20,760 \\
(18,815-23,914)\end{array}$ & $\begin{array}{c}\$ 60 \\
(50-112)\end{array}$ & $\begin{array}{c}346 \\
(185-429)\end{array}$ \\
\hline $\mathrm{RCV}$ & $\begin{array}{c}2 \\
(0-5)\end{array}$ & $\begin{array}{c}\$ 18 \\
(14-40)\end{array}$ & $\begin{array}{c}\$ 5 \\
(4-12)\end{array}$ & $\begin{array}{c}4 \\
(2-7)\end{array}$ & $\begin{array}{c}\$ 119 \\
(91-263)\end{array}$ & $\begin{array}{c}\$ 25 \\
(21-45)\end{array}$ & $\begin{array}{c}5 \\
(3-10)\end{array}$ \\
\hline MenA & $\begin{array}{c}2 \\
(0-5)\end{array}$ & $\begin{array}{c}\$ 3 \\
(2-5)\end{array}$ & $\begin{array}{c}\$ 1 \\
(1-4)\end{array}$ & $\begin{array}{c}3 \\
(1-4)\end{array}$ & $\begin{array}{c}\$ 15 \\
(11-27)\end{array}$ & $\begin{array}{c}\$ 4 \\
(3-10)\end{array}$ & $\begin{array}{c}3 \\
(1-7)\end{array}$ \\
\hline YFV & $\begin{array}{c}26 \\
(4-90)\end{array}$ & $\begin{array}{c}\$ 275 \\
(222-429)\end{array}$ & $\begin{array}{c}\$ 4 \\
(4-15)\end{array}$ & $\begin{array}{c}62 \\
(20-51)\end{array}$ & $\begin{array}{c}\$ 1727 \\
(1338-2771)\end{array}$ & $\begin{array}{c}\$ 22 \\
(16-53)\end{array}$ & $\begin{array}{c}77 \\
(33-136)\end{array}$ \\
\hline
\end{tabular}




\begin{tabular}{|c|c|c|c|c|c|c|c|}
\hline & Benefit-risk & & COI & & & VSLY & \\
\hline Vaccine & $\begin{array}{l}\text { COVID-19 } \\
\text { deaths) }\end{array}$ & $\begin{array}{c}\text { VPD } \\
\text { (millions) }\end{array}$ & $\begin{array}{c}\text { COVID-19 } \\
\text { (millions) }\end{array}$ & $\mathrm{BCR}(\mathrm{COI})$ & VPD (millions) & $\begin{array}{c}\text { COVID-19 } \\
\text { (millions) }\end{array}$ & $\begin{array}{c}\text { BCR } \\
(\mathrm{VSLY})\end{array}$ \\
\hline MCV2 (EPI-3) & $\begin{array}{c}13 \\
(2-44)\end{array}$ & $\begin{array}{c}\$ 63 \\
(56-73)\end{array}$ & $\begin{array}{c}\$ 7 \\
(5-14)\end{array}$ & $\begin{array}{c}10 \\
(4-13)\end{array}$ & $\begin{array}{c}\$ 493 \\
(427-601)\end{array}$ & $\begin{array}{c}\$ 36 \\
(27-74)\end{array}$ & $\begin{array}{c}14 \\
(7-19)\end{array}$ \\
\hline $\begin{array}{l}\text { EPI-1 (HepB3, Hib3, } \\
\text { PCV3, RotaC) }\end{array}$ & $\begin{array}{c}80 \\
(13-262)^{\mathrm{a}}\end{array}$ & $\begin{array}{c}\$ 1597 \\
(1521-1840)\end{array}$ & $\begin{array}{c}\$ 35 \\
(33-81)\end{array}$ & $\begin{array}{c}46 \\
(21-51)\end{array}$ & $\begin{array}{c}\$ 11,230 \\
(10,478-13,015)\end{array}$ & $\begin{array}{c}\$ 179 \\
(150-320)\end{array}$ & $\begin{array}{c}63 \\
(36-79)\end{array}$ \\
\hline $\begin{array}{l}\text { EPI-2 (MCV1, RCV1, } \\
\text { MenA, YFV) }\end{array}$ & $\begin{array}{c}112 \\
(18-372)\end{array}$ & $\begin{array}{c}\$ 3290 \\
(3089-3704)\end{array}$ & $\begin{array}{c}\$ 12 \\
(11-28)\end{array}$ & $\begin{array}{c}282 \\
(121-302)\end{array}$ & $\begin{array}{c}\$ 22,621 \\
(20,775-26,091)\end{array}$ & $\begin{array}{c}\$ 60 \\
(50-113)\end{array}$ & $\begin{array}{c}377 \\
(202-473)\end{array}$ \\
\hline EPI (all vaccines) & $\begin{array}{c}82 \\
(13-265)^{\mathrm{a}}\end{array}$ & $\begin{array}{c}\$ 4949 \\
(4776-5480)\end{array}$ & $\begin{array}{c}\$ 53 \\
(50-121)\end{array}$ & $\begin{array}{c}94 \\
(42-102)\end{array}$ & $\begin{array}{c}\$ 34,344 \\
(32,554-38,593)\end{array}$ & $\begin{array}{c}\$ 275 \\
(230-506)\end{array}$ & $\begin{array}{c}125 \\
(69-155)\end{array}$ \\
\hline
\end{tabular}

Note: BSRs comparing vaccine-preventable deaths to COVID-19 deaths were extracted from Abbas et al. (2020). ${ }^{10}$

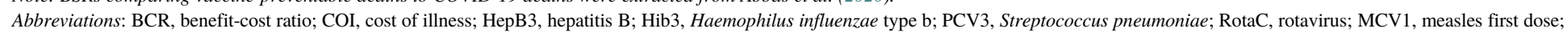
MCV2, measles second dose; RCV, rubella; MenA, Neisseria meningitidis serogroup A; VSLY, value of statistical life year.

${ }^{a}$ Includes averted deaths from diphtheria, tetanus, and pertussis, not included in the economic analysis. 
comprise $0.3 \%$ of the COI, with productivity loss due to death making up the remaining $99.7 \%$ of the COI. Estimated transportation costs and caregiver wages lost were negligible $(<0.1 \%)$. Sustaining immunization would generate $\$ 34,344$ million $(\$ 32,554-38,593)$ in the value of lives saved using the VSLY approach. Estimates using the VSL approach can be found in Appendix 5 in the Supplementary Material. Country- and vaccine-specific estimates for each approach are in Appendices 6-8 in the Supplementary Material.

Excess deaths from COVID-19 would result in \$53 million (\$50-\$121) of economic costs using the COI approach. Treatment costs comprise $22.8 \%$ of the total COI from COVID-19, with productivity loss making up the remaining 77.2\%. As with the VPD estimates, transportation costs and caregiver wages lost were negligible $(<0.1 \%)$. Using the VSLY approach, we anticipate COVID-19 would lead to \$275 million (\$230-506) in lost economic value.

The BCR using the COI approach ranged from 1:1 (1-2) for hepatitis B and MenA to 257:1 (107-275) for MCV1 (Figure 1). Across all vaccines, the BCR was 94:1 (42-102), compared with the benefit-risk ratio of $82: 1$, comparing numbers of vaccine-preventable deaths to those of COVID-19 deaths.

As expected, the VSLY approach generated higher BCRs. The lowest ratios using this approach were estimated for hepatitis B and MenA, with 2:1 (1-4) and 3:1 (1-7), respectively; the MCV1 generated the highest BCR of 346:1 (185-429). The overall BCR for routine immunization using VSLY was 125:1 (69-155).

One-way sensitivity analysis showed that the BCR estimated using the COI approach is sensitive to the number of deaths from VPDs and COVID-19 (Figure 2). Facility costs, careseeking rates, and ICU admittance also contribute notably to uncertainty in the BCR. Higher facility costs increased estimated COVID-19 costs more than costs due to VPDs and decreased the BCR.

The BCR calculated from the VSLY approach is sensitive to uncertainty in the deaths from VPDs and COVID-19, but uncertainty in GDP per capita had little impact on the estimate (Figure 3). The impact of each parameter on the estimates on the economic costs of

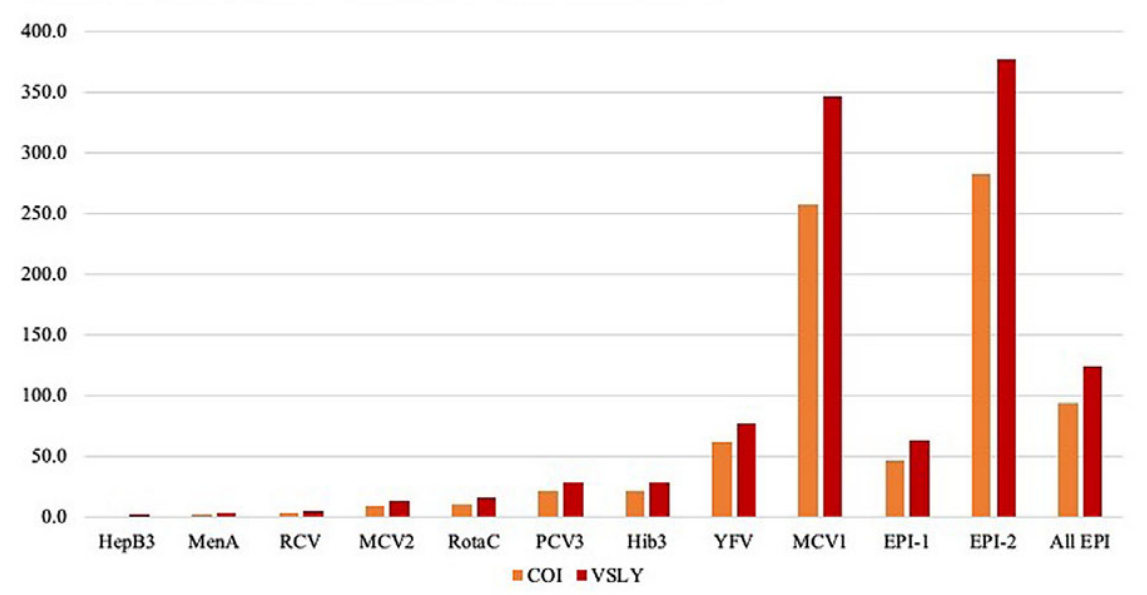

Figure 1. Benefit-risk ratios by vaccination using the cost of illness and value of statistical life-year approaches. 


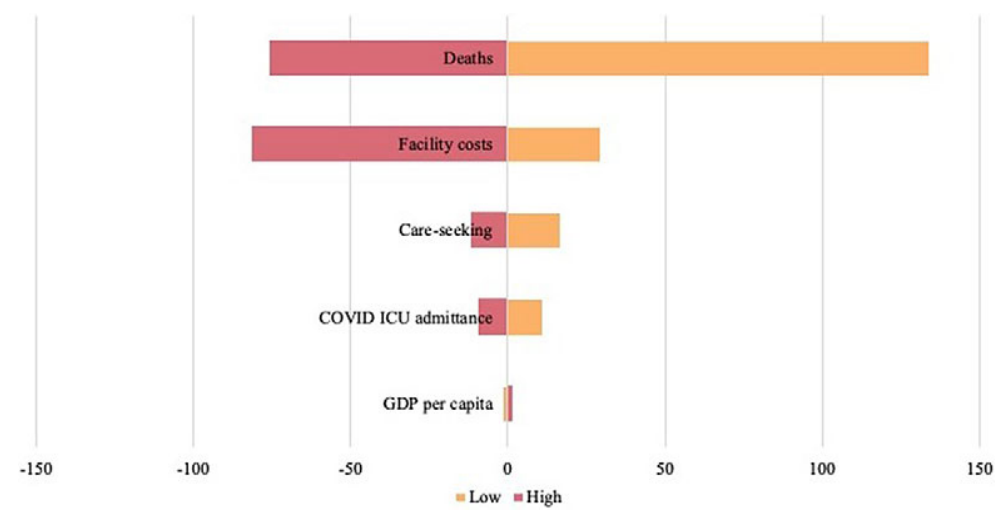

Figure 2. Sensitivity analysis for benefit-cost ratios using the cost of illness approach.

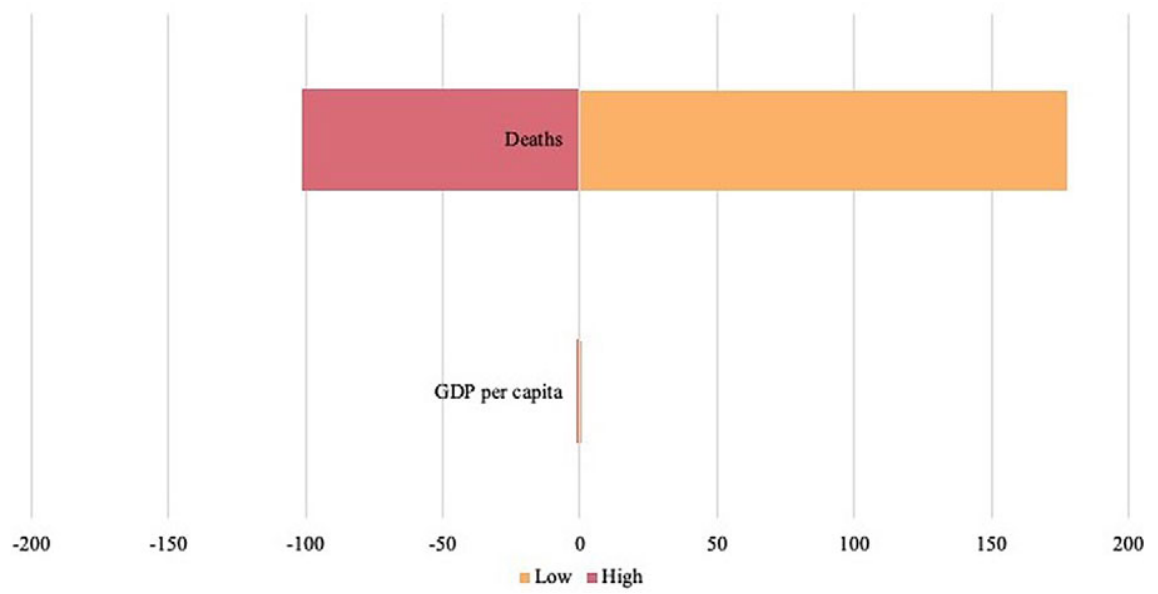

Figure 3. Sensitivity analysis for benefit-cost ratios using the value of statistical life-year approach.

vaccine-preventable deaths and COVID deaths are shown in Appendix 9 in the Supplementary Material.

Results from the scenario analysis that include costs of providing routine immunization during the pandemic are presented in Table 2. Using the COI approach, the lowest vaccinespecific BCR was for hepatitis B, which was $0(0-0)$ for all estimates that included costs of routine immunization. Measles first dose generated the highest vaccine-specific BCRs, ranging from 52 (40-53) in the reference case, 44 (35-44) with low costs of providing routine immunization during the pandemic, to 17 (16-17) with high costs of routine immunization. Using the VSLY approach, vaccine-specific BCRs ranged from 1 (1-1) for all hepatitis B estimates to 196 (131-220), 178 (123-197), and 94 (76-99) for measles first dose, using the reference case, low- and high-cost estimates, respectively. The BCRs for each EPI visit using the highest cost estimates for continuing routine immunization during the pandemic each show 
Table 2. BCRs of sustained routine immunization in Africa including the costs of routine immunization (95\% confidence intervals in parentheses).

\begin{tabular}{|c|c|c|c|c|c|c|}
\hline \multirow[b]{2}{*}{ Vaccine } & \multicolumn{3}{|c|}{ COI } & \multicolumn{3}{|c|}{ VSLY } \\
\hline & $\begin{array}{c}\text { BCR } \\
\text { including } \\
\text { immunization } \\
\text { costs } \\
\text { (reference) }\end{array}$ & $\begin{array}{l}\text { BCR including } \\
\text { immunization } \\
\text { costs with } \\
\text { mitigation } \\
\text { measures (low) }\end{array}$ & $\begin{array}{l}\text { BCR including } \\
\text { immunization } \\
\text { costs with } \\
\text { mitigation } \\
\text { measures (high) }\end{array}$ & $\begin{array}{c}\text { BCR } \\
\text { including } \\
\text { immunization } \\
\text { costs } \\
\text { (reference) }\end{array}$ & $\begin{array}{l}\text { BCR including } \\
\text { immunization } \\
\text { costs with } \\
\text { mitigation } \\
\text { measures (low) }\end{array}$ & $\begin{array}{l}\text { BCR including } \\
\text { immunization } \\
\text { costs with } \\
\text { mitigation } \\
\text { measures (high) }\end{array}$ \\
\hline HepB3 & $0(0-0)$ & $0(0-0)$ & $0(0-0)$ & $1(1-2)$ & $1(1-2)$ & $1(1-1)$ \\
\hline Hib3 & $6(4-6)$ & $5(4-5)$ & $2(2-2)$ & $19(12-22)$ & $18(12-20)$ & $10(8-11)$ \\
\hline PCV3 & $2(1-2)$ & $1(1-1)$ & $0(0-0)$ & $9(7-9)$ & $7(6-8)$ & $3(3-3)$ \\
\hline RotaC & $1(1-1)$ & $1(1-1)$ & $0(0-0)$ & $6(4-6)$ & $5(4-6)$ & $2(2-2)$ \\
\hline MCV1 & $52(40-53)$ & $44(35-44)$ & $17(16-17)$ & $\begin{array}{c}196(131- \\
220)\end{array}$ & $178(123-197)$ & $94(76-99)$ \\
\hline $\mathrm{RCV}$ & $2(2-2)$ & $2(2-2)$ & $1(1-1)$ & $8(5-10)$ & $8(5-9)$ & $4(3-5)$ \\
\hline MenA & $0(0-0)$ & $0(0-0)$ & $0(0-0)$ & $1(1-2)$ & $1(1-1)$ & $0(0-1)$ \\
\hline YFV & $0(0-0)$ & $0(0-0)$ & $0(0-0)$ & $2(1-2)$ & $2(1-2)$ & $1(1-1)$ \\
\hline MCV2 (EPI-3) & $9(7-10)$ & $8(6-8)$ & $3(3-3)$ & $36(22-46)$ & $32(21-40)$ & $16(12-17)$ \\
\hline $\begin{array}{l}\text { EPI-1 (HepB3, Hib3, } \\
\text { PCV3, RotaC) }\end{array}$ & $3(3-3)$ & $2(2-2)$ & $1(1-1)$ & $15(13-16)$ & $13(11-14)$ & $5(5-5)$ \\
\hline $\begin{array}{l}\text { EPI-2 (MCV1, RCV1, } \\
\text { MenA, YFV) }\end{array}$ & $13(12-13)$ & $11(10-11)$ & $4(4-4)$ & $75(64-78)$ & $63(55-65)$ & $25(23-25)$ \\
\hline EPI (all vaccines) & $66(36-70)$ & $62(34-66)$ & $38(25-39)$ & $116(66-141)$ & $114(66-138)$ & $97(60-115)$ \\
\hline
\end{tabular}

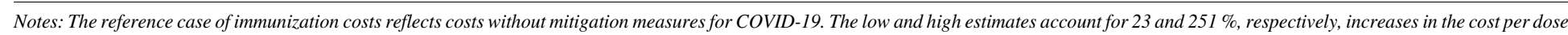
for implementing COVID-19 mitigation measures (Banks et al., 2021).

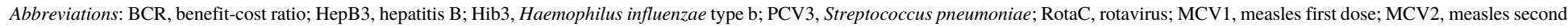
dose; RCV, rubella; MenA, Neisseria meningitidis serogroup A. 
benefits exceed costs. Using the highest estimate of costs, the BCR across all vaccines was 38 (25-39) using the COI approach and 97 (60-115) using the VSLY approach.

\section{Discussion}

This analysis demonstrates the significant economic costs associated with suspending routine immunization during the COVID-19 pandemic. Given the large number of VPDs with significant disease burden in Africa and the drastically different age distribution of disease burden from existing VPDs and COVID-19, the benefits of sustaining immunization services far exceed the risks due to COVID-19 in both health and economic terms. This is particularly true for measles, a highly infectious VPD with a high case-fatality rate among young children, which exhibited BCRs above 100:1 and as high as 346:1 in our analysis.

BCRs help weigh the benefits and costs associated with sustaining routine immunization. Of all the vaccines modeled, only hepatitis B and MenA exhibit BCRs where lower bounds of the $95 \%$ confidence intervals fall below 1:1 in the primary results. All estimates of the mean BCRs that compare costs of death are greater than 1:1, demonstrating that the economic impact of sustained routine immunization outweigh the risks of COVID-19 associated with immunization activities. Furthermore, the COI and VSLY BCRs are higher than the benefit-risk ratios that compare the number of deaths (Abbas et al., 2020). This is because the COI and VSLY approaches account for the relative ages of those impacted by VPDs compared to COVID-19. Using the COI approach, child deaths are associated with larger productivity loss compared with adults. Likewise, the VSLY approach assigns value to lives saved based on the remaining life expectancy at the age of death. For children, this yields a higher economic value.

The estimated BCRs from each EPI visit were high, ranging from 46:1 to 282:1 using the COI approach, and 63:1 to 377:1 using the VSLY approach. The high BCRs from EPI visits, during which children receive multiple vaccine doses at once, demonstrate that bundling the health benefits from immunization also minimizes the risk of COVID-19 transmission.

The scenario analysis that accounted for costs of providing immunization during the pandemic reaffirms that benefits of routine immunization outweigh the risk of increased COVID-19 transmission. Despite the potential cost increases of providing personal protective equipment, increasing hygiene practices, social distancing, and other measures, immunization remains an important tool for maintaining child health and preventing outbreaks of VPDs.

Using the COI approach, productivity loss comprises most of the economic cost of VPDs and COVID-19; however, treatment costs were also a significant driver of COVID-19 costs. Treating COVID-19 patients requires substantial personal protective equipment for healthcare workers, which drives up costs (Barasa \& Kairu, 2020). Emerging evidence suggests that hospitalized COVID-19 patients often have long inpatient stays, ranging from 4 to 21 days for patients who died in the hospital, and longer for patients discharged alive (Rees et al., 2020). Prolonged inpatient stays for COVID-19 patients may strain hospital capacity in low-resource settings. In the case of disrupted immunization, simultaneous outbreaks of VPDs and COVID-19 could place a synergistic burden on the hospital resources.

These economic implications concur with existing evidence that suspending, or reducing, routine immunization activities will result in health consequences more catastrophic than COVID-19 transmission. To mitigate the effects of suspended immunization services, 
countries must address supply- and demand-side factors contributing to the COVID-19related disruption. Countries responding to the WHO pulse survey indicated that prioritization of health services was an important step toward minimizing disruptions due to COVID-19 (World Health Organization, 2020c). Community outreach to inform the public of the importance of immunization, provide updates on service changes, and inform the public of safety precautions at immunization sites may help overcome demand-side challenges. This analysis demonstrates that implementing safety measures to reduce the risk of COVID-19 transmission at the vaccination sites would be justified even if they increase costs of immunization by more than $250 \%$. The ability to adapt in the wake of the COVID-19 pandemic will help sustain a resilient immunization delivery system in the face of potential future pandemics or other enduring crises.

This analysis has several limitations. This analysis is based on a model estimating the benefits and risks associated with a 6-month suspension in immunization services (Abbas et al., 2020). The duration and extent of service disruption vary between countries, which could contribute substantially to uncertainty in expected excess deaths. We attempted to address this in sensitivity analysis by using an estimated range of deaths for VPDs and COVID-19 (Abbas et al., 2020). Additional country surveys of the impact of COVID-19 on health service delivery may better inform future modeling studies.

This analysis excludes the economic impact of preventing nonfatal illness caused by COVID-19 and childhood vaccine-preventable illness. Morbidity from COVID-19 and vaccine-preventable diseases may impact productivity loss, and it is unknown whether nonfatal cases of COVID-19 or vaccine-preventable diseases would be more costly. Prior studies on economic benefits of immunization suggest that costs of death comprise the majority of the economic burden of disease (Watts et al., 2021), which served the basis of this analysis. The scenario analysis that includes the cost of providing routine immunization during COVID-19 accounts for costs of implementing mitigation measures. If mitigation measures effectively reduce risk of COVID-19 transmission during immunization visits, these estimates will conservatively reflect the benefit of continuing routine immunization during the pandemic. Lastly, this analysis only considers COVID-19 risks to household members and not to health workers who may be involved with both treating COVID-19 and carrying out immunization services. It also does not account for health workforce supply constraints that may arise if health systems are significantly overburdened with COVID-19 cases and exhibit limited capacity to direct efforts toward other service provision, including primary care and immunization services.

Lastly, these estimates are based on direct benefits of vaccination and COVID-19 cases transmitted during immunization visits. Both measles and COVID-19 are highly transmissible and may lead to outbreaks in communities with low immunity (Ferrari et al., 2008; Garba et al., 2020). Continuing routine immunization with mitigation measures to prevent COVID-19 transmission will be important for reducing the risk of COVID-19 and measles outbreaks in low-resource settings.

In conclusion, estimates of child deaths caused by halting routine immunization activities suggest that the benefits of continuing immunization far outweigh the risk of COVID-19 deaths among households visiting immunization sites. BCRs provide additional insight for vaccine advocates and country governments in the African region, as they prioritize health services during the COVID-19 pandemic. Despite increased costs of continuing immunization services during the pandemic, preventing childhood deaths from 
VPDs should be prioritized. Our analysis also demonstrates both from a health and economic perspective that if delivery of only one vaccine should be prioritized to be maintained, uninterrupted, during the COVID-19, it should be measles first dose, which consistently exhibited BCRs above 100:1. Countries should immediately prioritize ways to continue the delivery of measles vaccine and catch up on forgone services as quickly as possible to prevent potentially large future health and economic consequences due to measles outbreaks.

Acknowledgments. We are grateful for the cooperation and guidance from Mark Jit, Kaja Abbas, Stefan Flasche, Simon Proctor, and the CMMID COVID-19 Working Group at the London School of Hygiene \& Tropical Medicine.

Competing interests. The authors declare no competing interests.

Supplementary Materials. To view supplementary material for this article, please visit http://doi.org/10.1017/ bca.2021.13.

\section{References}

Abbas, Kaja, Simon R. Procter, Kevin van Zandvoort, Andrew Clark, Sebastian Funk, Tewodaj Mengistu, Dan Hogan, et al. 2020. "Routine Childhood Immunisation During the Covid-19 Pandemic in Africa: A Benefit-Risk Analysis of Health Benefits Versus Excess Risk of Sars-Cov-2 Infection.” The Lancet Global Health, 8: e1264-e1272.

Andre, Francis E., Robert Booy, Hans L. Bock, John Clemens, Sanjoy K. Datta, T. Jacob John, Bee Wah Lee, et al. 2008. "Vaccination Greatly Reduces Disease, Disability, Death and Inequity Worldwide." Bulletin of the World Health Organization, 86(2): 140-146.

Banks, Christina, Allison Portnoy, Flavia Moi, Laura Boonstoppel, and Stephen C. Resch 2021. "Cost of Vaccine Delivery Strategies in Low- and Middle-Income Countries During the COVID-19 Pandemic.” Vaccine, 39(35): 5046-5054. https://doi.org/10.1016/J.VACCINE.2021.06.076.

Barasa, Edwine and Angela Kairu. 2020. "What Does It Cost to Treat a Covid-19 Patient in Kenya?" KEMRI. Available at https://kemri-wellcome.org/zp-content/uploads/2020/07/HERU-Policy-brief-Treatment-costs-forCOVID-19-patients-in-Kenya.pdf (accessed July 17, 2020).

Bishai, David, Benjamin Johns, Divya Nair, Juliet Nabyonga-Orem, Braka Fiona-Makmot, Emily Simons, and Alya Dabbagh. 2011. "The Cost-Effectiveness of Supplementary Immunization Activities for Measles: A Stochastic Model for Uganda." Journal of Infectious Disease, 204: S107-S115.

Davies, Nicholas G., Sedona Sweeney, Sergio Torres-Rueda, Fiammetta Bozzani, Nichola Kitson, Edwine Barasa, Simon Procter, et al. 2020. "The Impact of Coronavirus Disease 2019 (Covid-19) on Health Systems and Household Resources in Africa and South Asia." Preprint published May 8, 2020. medRxiv. https://doi.org/ 10.1101/2020.05.06.20092734.

de Broucker, Gatien, So Yoon Sim, Logan Brenzel, Margaret Gross, Bryan Patenaude, and Dagna O. Constenla. 2020. "Cost of Nine Pediatric Infectious Illnesses in Low- and Middle-Income Countries: A Systematic Review of Cost-of-Illness Studies." PharmacoEconomics, 38(10): 1071-1094.

Demographic and Health Survey Program. n.d. "Demographic and Health Surveys." DHS. Available at https:// dhsprogram.com/data/ (accessed August 12, 2020).

Ferrari, Matthew J., Rebecca F. Grais, Nita Bharti, Andrew J.K. Conlan, Ottar N. Bjørnstad, Lara J. Wolfson, Philppe J. Guerin, Ali Djibo, and Bryan T. Grenfell. 2008. "The Dynamics of Measles in Sub-Saharan Africa." Nature, 451(7179): 679-684. https://doi.org/10.1038/nature06509.

Garba, S. M., J. M. S. Lubuma, and B. Tsanou. 2020. "Modeling the Transmission Dynamics of the COVID-19 Pandemic in South Africa." Mathematical Biosciences, 328: 108441. https://doi.org/10.1016/ J.MBS.2020.108441.

Global Burden of Disease Health Financing Collaborator Network. 2018. "Trends in Future Health Financing and Coverage: Future Health Spending and Universal Health Coverage in 188 Countries, 2016-40." Lancet (London, England), 391(10132): 1783-1798.

International Labor Organization. 2018. “ILOSTAT.” Geneva, Switzerland. Available at https://ilostat.ilo.org/data/ (accessed July 8, 2018). 
Kim, Sun-Young, Gene Lee, and Sue J. Goldie. 2010a. "Economic Evaluation of Pneumococcal Conjugate Vaccination in the Gambia." BMC Infectious Disease, 10(1): 260.

Kim, Sun-Young, Steve Sweet, David Slichter, and Sue J. Goldie. 2010b. "Health and Economic Impact of Rotavirus Vaccination in Gavi-Eligible Countries.” BMC Public Health, 10: 253.

Lee, Bruce Y., Diana L. Connor, Sarah B. Kitchen, Kristina M. Bacon, Mirat Shah, Shawn T. Brown, Rachel R. Bailey, Yongjua Laosiritaworn, Donald S. Burke, and Derek A. T. Cummings. 2011. "Economic Value of Dengue Vaccine in Thailand." The American Journal of Tropical Medicine and Hygiene, 84(5): 764-772.

Li, Xiang, Christinah Mukandavire, Zulma M. Cucunubá, Kaja Abbas, Hannah E. Clapham, Mark Jit, Hope L. Johnson, et al. 2019. "Estimating the Health Impact of Vaccination Against 10 Pathogens in 98 Low and Middle Income Countries from 2000 to 2030.” Preprint published August 27, 2019. medRxiv. https://doi.org/10.1101/ 19004358.

Ma, Xiya and Dominique Vervoort. 2020. "Critical Care Capacity During the Covid-19 Pandemic: Global Availability of Intensive Care Beds.” Journal of Critical Care, 58: 96-97.

Mosser, Jonathan F., William Gagne-Maynard, Puja C. Rao, Aaron Osgood-Zimmerman, Nancy Fullman, Nicholas Graetz, Roy Burstein, et al. 2019. "Mapping Diphtheria-Pertussis-Tetanus Vaccine Coverage in Africa, 2000-2016: A Spatial and Temporal Modelling Study.” Lancet (London, England), 393(10183): 1843-1855.

Parashar, Umesh D., Erik G. Hummelman, Joseph S. Bresee, Mark A. Miller, and Roger I. Glass. 2003. "Global Illness and Deaths Caused by Rotavirus Disease in Children.” Emerging Infectious Diseases, 9(5): 565-572.

Rees, Eleanor M., Emily S. Nightingale, Yalda Jafari, Naomi Waterlow, Samuel Clifford, Carl A. B. Pearson, Thibaut Jombert, Simon R. Procter, and Gwenan M. Knight. 2020. "Covid-19 Length of Hospital Stay: A Systematic Review and Data Synthesis.” BMC Medicine, 18: 270.

Robinson, Lisa A., James K. Hammitt, Michele Cecchini, Kalipso Chalkidou, Karl Claxton, Maureen Cropper, Patrick Hoang-Vu Eozenou, et al. 2019a. "Reference Case Guidelines for Benefit-Cost Analysis in Global Health and Development." Available at https:/cdn1.sph.harvard.edu/wp-content/uploads/sites/2447/2019/05/ BCA-Guidelines-May-2019.pdf (accessed July 17, 2020).

Robinson, Lisa A., James K. Hammitt, and Lucy O'Keeffe. 2019b. "Valuing Mortality Risk Reductions in Global Benefit-Cost Analysis.” Journal of Benefit-Cost Analysis, 10(S1): 15-50.

Rudan, Igor, Lana Tomaskovic, Cynthia Boschi-Pinto, Harry Campbell, and WHO Child Health Epidemiology Reference Group. 2004. "Global Estimate of the Incidence of Clinical Pneumonia Among Children Under Five Years of Age." Bulletin of the World Health Organization, 82(12): 895-903.

Sim, So Yoon, Elizabeth Watts, Dagna Constenla, Logan Brenzel, and Bryan N. Patenaude 2020. "Return on Investment from Immunization Against 10 Pathogens in 94 Low- and Middle-Income Countries, 2011-30." Health Affairs, 39(8): 1343-1353. https://doi.org/10.1377/hlthaff.2020.00103.

Sinha, Anushua, Dagna Constenla, Juan Esteban, Rosalyn O’Loughlin, Elizabeth Gomez, Fernando de la Hoz, Maria Teresa Valenzuela, and Ciro A. de Quadros. 2008. "Cost-Effectiveness of Pneumococcal Conjugate Vaccination in Latin America and the Caribbean: A Regional Analysis." Revista Panamericana de Salud Pública, 24(5):304-313.

Sriudomporn, Salin, Elizabeth Watts, So Yoon Sim, Raymond Hutubessy, and Bryan N. Patenaude. 2021. "Cost Estimates of Immunization Agenda 2030 in 194 WHO Member States." Unpublished.

Stenberg, Karin, Jeremy A. Lauer, Georgios Gkountouras, Christopher Fitzpatrick, and Anderson Stanciole. 2018. "Econometric Estimation of Who-Choice Country-Specific Costs for Inpatient and Outpatient Health Service Delivery." Cost Effectiveness and Resource Allocation, 16: 11.

The World Bank. 2019. "The World Bank Databank: GDP Per Capita (Current US\$)." Available at https:// data.worldbank.org/indicator/NY.GDP.PCAP.CD (accessed March 18, 2019).

U.S. Department of State. 2017. "Country Reports on Human Rights Practices." Available at https://www.state. gov/reports/2017-country-reports-on-human-rights-practices/ (accessed March 18, 2019).

United Nations. 2019. "World Population Prospects - Population Division - United Nations.” Available at https:// population.un.org/wpp/Download/Standard/Mortality/ (accessed July 31, 2021).

Wang, Dawei, Bo Hu, Chang Hu, Fangfang Zhu, Xing Liu, Jing Zhang, Binbin Wang, et al. 2020. "Clinical Characteristics of 138 Hospitalized Patients with 2019 Novel Coronavirus-Infected Pneumonia in Wuhan, China." Journal of the American Medical Association, 323(11): 1061-1069.

Watts, Elizabeth, So Yoon Sim, Dagna Constenla, Salin Sriudomporn, Logan Brenzel, and Bryan Patenaude. 2021. "Economic Benefits of Immunization for 10 Pathogens in 94 Low- and Middle-Income Countries from 2011 to 
2030 Using Cost-of-Illness and Value-of-Statistical-Life Approaches." Value in Health: The Journal of the International Society for Pharmacoeconomics and Outcomes Research, 24(1): 78-85. https://doi.org/10.1016/ J.JVAL.2020.07.009. World.

World Health Organization. 2020a. "Covid-19 Cases Top 10,000 in Africa." Geneva, Switzerland: World Health Organization. Available at https://www.afro.who.int/news/covid-19-cases-top-10-000-africa (accessed July 17, 2020).

World Health Organization. 2020b. "Maintaining Essential Health Services: Operational Guidance for the Covid19 Context.” Geneva, Switzerland: World Health Organization. Available at https://www.who.int/publications/ i/item/WHO-2019-nCoV-essential-health-services-2020.1 (accessed July 17, 2020).

World Health Organization. 2020c. "Pulse Survey on Continuity of Essential Health Services During the Covid-19 Pandemic [Interim Report].” Edited by World Health Organization. Geneva, Switzerland: World Health Organization.

World Health Organization. 2020d. "WHO and UNICEF Warn of a Decline in Vaccinations During Covid-19." Geneva, Switzerland: World Health Organization. Available at https://www.who.int/news-room/detail/15-072020-who-and-unicef-warn-of-a-decline-in-vaccinations-during-covid-19\#: :text=GENEVA\%2FNEW\% 20YORK\%2C\%2015\%20July,by\%20the\%20COVID\%2D19\%20pandemic (accessed July 17, 2020).

World Health Organization. 2020e. "WHO Director-General's Opening Remarks at the Media Briefing on COVID19 - 11 March 2020.” Geneva, Switzerland: World Health Organization. Available at https://www.who.int/dg/ speeches/detail/who-director-general-s-opening-remarks-at-the-media-briefing-on-covid-19_-11-march-2020 (accessed July 17, 2020).

World Health Organization and UNICEF. 2020. "Immunization Coverage: Are We Losing Ground?” Edited by World Health Organization and UNICEF.

Cite this article: Watts, E, Mak, J. and Patenaude, B. 2022. "Benefit-Cost Ratios of Continuing Routine Immunization During the COVID-19 Pandemic in Africa." Journal of Benefit-Cost Analysis 13: 91-106, doi:10.1017/bca.2021.13 